\title{
Animal personality due to social niche specialisation
}

\author{
Ralph Bergmüller ${ }^{1}$ and Michael Taborsky ${ }^{2}$
}

\author{
${ }^{1}$ Department of Eco-Ethology, Institute of Biology, University of Neuchâtel, Rue Emile Argand 11, CH-2009 Neuchâtel, Switzerland \\ ${ }^{2}$ Department of Behavioural Ecology, Institute of Ecology and Evolution, University of Bern, Wohlenstr. 50a, CH-3032 \\ Hinterkappelen, Switzerland
}

\begin{abstract}
The existence of 'animal personality', i.e. consistent individual differences in behaviour across time and contexts, is an evolutionary puzzle that has recently generated considerable research interest. Although social factors are generally considered to be important, it is as yet unclear how they might select for personality. Drawing from ecological niche theory, we explore how social conflict and alternative social options can be key factors in the evolution and development of consistent individual differences in behaviour. We discuss how animal personality research might benefit from insights into the study of alternative tactics and illustrate how selection can favour behavioural diversification and consistency due to fitness benefits resulting from conflict reduction among social partners.
\end{abstract}

\section{The evolution of animal personality}

Within species or populations, individuals often vary consistently in their responses to environmental and social challenges, such as how to find food, deal with predators, or compete with conspecifics. Moreover, consistent individual differences in behaviour are often correlated across functional contexts. Such 'personalities', 'behavioural syndromes' or 'coping styles' are apparently ubiquitous in animals, including humans [1-7], but their ultimate causes are still an evolutionary puzzle (Box 1).

A number of hypotheses have been proposed to explain animal personality, either focusing on potential constraints or on adaptive causes $[2,5,8,9]$. While ecological factors, such as the influence of predation, have been proposed as important causes of personality variation within populations [10], the potential significance of social factors has received less attention (but see [11-14]). Here we propose that social conflict and alternative social options are of key importance for the development and evolution of animal personality. Drawing from an analogy to the ecological niche, we outline how within-species competition for particular social niches can select for consistent individual differences among conspecifics. First, we illustrate how the concept of the ecological niche can help in understanding consistent behavioural variation among members of a population. Second, we explain why social conflict and the existence of alternative social niche options can be key factors involved in the generation of animal personality. Third, we highlight how consistent individual differences in behaviour can be adaptive due to the reduction in conflict costs while saving the costs of switching between social niches. Fourth, we outline how specialisation by choosing a certain niche can affect the expression of behaviours in other contexts, thereby potentially generating behavioural syndromes. Fifth, we discuss the significance of developmental plasticity for the generation of adaptive personality differences. Sixth, we briefly review the evidence for the influence of social experience on variation in personality among members of a population, before we finally argue that similar mechanisms might be involved in producing trait variation in animal personality and alternative behavioural tactics.

\section{The intra-specific ecological niche}

The concept of the ecological niche is a cornerstone of ecological theory $[15,16]$. It suggests that the ecology of individuals or species with similar requirements (i.e. niches) is adjusted by 'niche differentiation' when they occur sympatrically, thereby reducing conflict resulting

\footnotetext{
Glossary

Animal personality: consistent differences between individuals in their behaviour across time and contexts [58].

Behavioural consistency: the behaviour of individuals remains stable over time in the sense that each individual does not express the full range of behavioural trait values present in its population [58]. This is reflected in an intra-individual correlation when subjects are measured repeatedly in the same context. As consistency is a relative measure applying to inter-individual comparisons it has also been referred to as 'differential consistency' [60]. Character displacement: a niche shift of morphological, ecological, behavioural or physiological traits in individuals of sympatric species caused by competition [79]. Here we apply this concept to niche diversification of behavioural phenotypes among interacting conspecifics that result from competition for the same social or ecological niche.

Ecological niche: the conditions and resources needed by an individual or species in order to practice its way of life $[15,16]$.

Social conflict: an interaction among conspecifics reflecting conflicting fitness interests that is typically costly for the involved parties, because individuals act on incompatible goals or interests [80]. Social conflicts result, for instance, from competition for access to food, mates or other resources.

Social niche: the social conditions an individual needs to practice its way of life. These conditions are shaped by interactions with conspecifics. Typically, individuals can choose between different 'social niche options' or 'social roles' when adopting a social niche.

Social role: the realised behaviour or tactic an individual uses in response to social challenges such as competing for food, space or mating partners. This is equivalent to the 'realised niche' in ecological theory [16]. Examples for social roles include dominant vs. subordinate positions in a hierarchy, philopatry vs. dispersal, producing vs. scrounging, and different functions when tasks are shared in cooperative species with division of labour. An inflexible status the individual cannot choose or change, such as age, sex and life history state (e.g. male, female, parent and offspring) are not considered 'social roles'.

Social niche specialisation: a consistently different way to behave relative to other individuals in a group or population resulting from choice of a particular social role.
} 


\section{Box 1. Three evolutionary riddles concerning animal personality}

Firstly, behaviour is generally expected to be flexible rather than consistent over time in order to allow appropriate responses to variation in environmental and social conditions. In contrast to this expectation, many studies have shown that behaviour is often remarkably consistent within individuals over time [2,5]. This is apparent especially if the behaviour of individuals relative to one another is concerned. In other words, the behaviour remains stable over time in the sense that each individual expresses only a part of the behavioural trait values present in a population, even if the absolute level of behaviour of an individual somewhat fluctuates [13]. Secondly, behavioural trait values of individuals in a population should evolve towards a mean optimal phenotype, i.e. evolved traits should show little variance. However, recent research has revealed that individuals in a population frequently differ substantially in their behavioural type $[1-3,5,51,81,82]$. Thirdly, independent behavioural responses should be favoured over behavioural correlations across functionally unrelated contexts, because uncoupled behaviours might allow for more adequate responses to specific challenges. In contrast to this prediction, a number of studies have shown that seemingly unrelated behaviours are often correlated among each other $[2,10,81,83-86]$. A typical example is the frequently found correlation between boldness and aggression, which can depend on ecological factors such as the presence of predators in a habitat [10].

from competition for food, space or other important resources that are limiting. In other words, because of competition for the same niche, different organisms 'push' each other away from their initial optimum by character displacement. Hence, an organism's ecology depends on the presence of other organisms with a similar or overlapping niche. Traditionally, the concept of the ecological niche has been employed to understand the evolution and ecology of between-species differences $[17,18]$. However, it has also been used as an analogy to describe between-individual differences within species or populations [19-22]. Particularly, the niche concept has been applied in the context of intra-specific specialisation in diet and habitat use [22], and as an analogy, of intraspecific social specialisation $[2,23,24]$. In the human personality literature, a situation in which different individuals in the same population create or encourage different sets of experiences for themselves has been coined 'niche construction' [25].

The ecological niche concept deals with the response of organisms to the distribution of resources and competitors, or in other words, with the mechanisms by which individuals of different species resolve a conflict over resources. Below, we review evidence for the idea that consistent individual differences in behaviours within populations, i.e. animal personality, can result from a similar process. We propose that between individual consistency and behavioural diversification in one or multiple behaviours can reflect individual specialisation in different social niches, thereby reducing the conflict for resources among conspecifics (Box 2).

\section{The social environment: a key factor for personality evolution}

The social environment is a crucial factor shaping behavioural traits and animal personality $[2,5,11-14]$. The dynamics of social evolution are comparable to those of host-parasite, predator-prey and interspecific mutualism interactions, because social traits are both targets and agents of selection [26]. However, a conceptual basis concerning the social factors involved in generating consistent individual differences in behavioural traits and correlations among different behaviours is so far missing. Here we suggest that (a) social conflict and (b) the simultaneous presence of different social niche options are key factors involved in the generation of animal personality.

(a) Social conflict is a ubiquitous evolutionary force, existing, for instance, between the sexes [27], among parents and offspring [28], between competitors for access to mates [29] and for resources such as food, space and shelter [30]. Given that conflict involves an increase in interaction costs between the involved parties, mechanisms

\section{Box 2. Components of social niche specialisation}

Social niche specialisation comprises four components. (1) Individuals try to adopt a certain social role (e.g. how to acquire resources), depending on their phenotype, which includes individual idiosyncrasies such as size, condition or experience. (2) Conflict over resources can lead to character displacement, i.e. individuals that cannot realise a preferred social niche shift to an alternative option. (3) The social role adopted by an individual can have long-lasting effects on its phenotype, because experience canalises the behaviour by positive feedback. (4) Selection favours diversification and consistency in behaviours if this results in reduced conflict. Some examples can illustrate this:

(1) Role choice. Empirical evidence suggests that individuals with particular behavioural tendencies adopt certain social niches. For instance, shy individuals (measured as exploration propensity in a novel environment) tended to be more gregarious when foraging than others in sheep (Ovis aries) [87]. In common lizards (Lacerta vivipara), independently measured variation of sociability of juveniles was associated with their dispersal behaviour [52].

(2) Character displacement. In great tits (Parus major), fast explorers (who also tend to be more aggressive) will only become dominant in a group when they are rare [13]. Explorative individuals failing to obtain a high rank are likely to end up in a low rank position, while intermediate positions are occupied by slow explorers.
(3) Temporal consistency. Social experience can cause long-lasting effects on behavioural phenotypes (Table 1). For instance, in a cooperatively breeding fish (Neolamprologus pulcher), the early post-larval social environment affects the social behaviours of juveniles [88]. Cross fostering experiments between two different species of tits (Parus major and Cyanistes caeruleus) resulted in lifelong feeding shifts of experimental subjects [89].

(4) Conflict reduction. Variation among social partners in personality traits can reduce the conflict potential and resulting fitness costs. Interactions between pigs in resident-intruder tests resulted in less injuries when one or both of two contestants were non-aggressive, compared to when both were aggressive [90]. In guppies (Poecilia reticulata), individuals in groups consisting of shy and bold types were more likely to feed successfully than individuals in groups composed only of either bold or shy individuals [91].

There is empirical evidence for niche preference, character displacement, long-term effects of social experience, and cost reduction by behavioural diversification. It remains to be established whether and how these components interact in the generation and maintenance of personality differences. 
Table 1. Experimental evidence suggesting social effects on personality and character displacement

\begin{tabular}{|c|c|c|c|c|c|c|}
\hline Species & $\begin{array}{l}\text { Social } \\
\text { environment }\end{array}$ & $\begin{array}{l}\text { Experimental } \\
\text { manipulation }\end{array}$ & $\begin{array}{l}\text { Context of } \\
\text { conflict }\end{array}$ & $\begin{array}{l}\text { Change in } \\
\text { behaviour }\end{array}$ & $\begin{array}{l}\text { Lasting effects } \\
\text { on behaviour }\end{array}$ & References \\
\hline 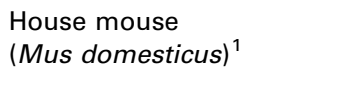 & Siblings & Sex ratio of litters & $\begin{array}{l}\text { Sibling } \\
\text { composition } \\
\text { (sex-ratio) }\end{array}$ & $\begin{array}{l}\text { Attack latency / } \\
\text { behavioural } \\
\text { flexibility }\end{array}$ & No / Yes & {$[63]$} \\
\hline Great tit (Parus major $)^{2}$ & Siblings & Food rationing & $\begin{array}{l}\text { Sibling } \\
\text { competition }\end{array}$ & $\begin{array}{l}\text { Exploration } \\
\text { behaviour }\end{array}$ & Yes & {$[64]$} \\
\hline $\begin{array}{l}\text { Guppy } \\
\text { (Poecilia reticulata) }\end{array}$ & Group & Rearing density & $\begin{array}{l}\text { Competition } \\
\text { for food }\end{array}$ & $\begin{array}{l}\text { Sociability } \\
\text { (shoaling } \\
\text { tendency) }\end{array}$ & Not measured & [94] \\
\hline $\begin{array}{l}\text { Cichlid } \\
\text { (Neolamprologus pulcher) }\end{array}$ & Group & $\begin{array}{l}\text { Rearing conditions } \\
\text { (with or without } \\
\text { dominants) }\end{array}$ & $\begin{array}{l}\text { Competition } \\
\text { for shelter }\end{array}$ & $\begin{array}{l}\text { Aggressive and } \\
\text { submissive } \\
\text { behaviours }\end{array}$ & Yes & [88] \\
\hline
\end{tabular}

${ }^{1}$ Selection line for long attack latency; ${ }^{2}$ Selection line for exploration

reducing the scope of conflict should be favoured by selection. Intrinsic individual differences in behaviour among social partners can reduce the scope for overt conflict, as illustrated, for instance, by the coexistence of producers and scroungers in resource acquisition [31-33]. Niche diversification of interacting behavioural phenotypes ('social character displacement') is hence a potential means of reducing the costs of conflict, particularly when conflict results from competition over resources.

(b) The social environment often provides different options to cope with a problem. This has been studied extensively in the context of alternative reproductive tactics, where intra- and inter-sexual selection can favour divergent behavioural and morphological types within a sex $[34,35]$. Alternative solutions to problems such as acquiring food or other resources might also generate diverging social niches, such as producers and scroungers in resource use [33], hawks and doves in contest situations [36], bold and shy explorers in a dispersal or foraging context [37], cooperative and selfish partners in social interactions [14], or individuals performing different tasks in cooperative societies [38]. The emergence of different social options for individuals of the same group or population results from (i) ecological parameters including the distribution of resources, such as food, shelter and breeding opportunities; and (ii) the social conditions, such as a hierarchy structure, variation in resource holding potential, or more generally, any spatial or temporal environmental variation allowing for alternative responses to a social challenge, thereby creating multiple social niches.

\section{Social conflict generating consistent individual differences}

Social conflict can select for the stable coexistence of different behavioural types, as has been shown with help of evolutionary game theory [36]. Negative frequency dependence, which means that the rare type has an advantage, can maintain alternative behavioural types in a population [11], because it reduces conflict and associated costs. For instance, if individuals can act either as producers (indi- viduals acquiring resources) or as scroungers (individuals exploiting resources made available by others), the scroungers will be more successful when they are rare compared to producers. Conversely, producers will be more successful when they become rare [33]. However, frequency dependence can maintain either a situation where individuals perform alternative behaviours with certain probabilities (i.e. their behaviour is not consistent), or where certain proportions of individuals pursue divergent strategies consistently [11]. The social niche concept predicts that consistent individual differences should be favoured over switching, because competition for niches makes the latter more costly (Box 2). For instance, switching is costly if alternative social roles are already occupied by others preventing social partners from using that niche; such as when owning a territory, reproducing within a group, or performing certain tasks in species with division of labour [39-41]. It is important to note that slight differences in individual idiosyncrasies might cause variation in pay-offs associated with social interactions. Hence, the adoption of an alternative role (e.g. if the preferred option is taken by others) might yield higher fitness as compared to engaging in costly contests. This is a much subtler and less conspicuous form of competition compared to what we usually associate with competition for social roles (e.g. overt conflict). For instance, evidence shows that individuals of the same species can reduce the amount of conflict by feeding on different types of food when competition for food is high $[22,42]$. Similarly, individuals might also reduce conflict by reducing social niche overlap. For instance, in normally solitary sweat bees, experimental nesting associations resulted in individuals specialised either on excavation or nest guarding [40]. In Table 1 we list examples suggesting that social interaction resulting from competition can cause a lasting modification of behavioural traits due to social character displacement.

In addition to the costs of conflict, switching to a different niche can require changes in physiology and behaviour of the individual, which can be costly [43-45]. Switching might become more and more constrained with increasing 
age if learning or physiological adjustments are required to adopt a new role. For example, for philopatric individuals specialised in resource defence in a known territory it might be costly to develop sensory abilities and behaviours needed to successfully explore alternative areas to which to disperse [46]. Additionally, learning a social tactic can increase the efficiency of using it [47]. Therefore, in contrast to the negative feedback mechanism favouring individuals that reduce the costs of conflict by adopting different social roles (character displacement and negative frequency dependence), a positive feedback mechanism can favour behavioural consistency of individuals due to the benefits of specialisation and the resulting costs of switching. Acting in concert these two feedback mechanisms might generate behavioural divergence and consistency.

\section{Social conflict generating behavioural correlations}

Combinations of traits that work well together can be favoured by correlational selection $[48,49]$. This has been suggested to also explain certain correlations of personality traits $[3,50,51]$. We propose two processes generating adaptive behavioural correlations across functional contexts.

(a) When social conflict causes individuals to occupy different environments, environmental correlations can select for particular trait combinations. For instance, intraspecific competition can yield different dispersal strategies, with some individuals avoiding high-density conditions and others accepting a high density [52]. If further ecological conditions, such as the distribution of food or other resources differ systematically for individuals pursuing these two strategies, the correlated ecological challenges can select for adaptive trait combinations.
With a graphical model we illustrate how social competition might generate the development of adaptive correlations between behaviours (Figure 1). Imagine resource competition among individuals and different social solutions that reduce this conflict, e.g. producer and scrounger strategies or hawk and dove tactics. Even between genetically identical individuals, small initial asymmetries will exist between them in state, capability, condition or experience, so that the options to deal with conflict depend on the individuals' intrinsic genetic quality, developmental differences, and random effects (such as their exposition to different microhabitats). Hence, selection should favour individuals specialising by adopting a particular niche (Box 2), which will reduce interaction costs and at the same time create consistent individual differences. Moreover, choosing a certain option in response to an environmental or social challenge will expose individuals to divergent environmental influences. Therefore, positive feedback based on experience with a certain role might select for, or stabilise the behavioural specialisation in several traits. When individuals act as producers, for instance because of their low competitive ability, this might affect how they deal with other challenges (Figure 1), e.g. the need to be explorative in order to find food, which will in turn increase predation risk and hence the demands to be vigilant. In contrast, scroungers might need to primarily focus on the behaviour of their conspecifics (potential producers) and develop strategies to efficiently exploit their effort. Overall, cascading effects following from social specialisation in one context might affect the behavioural options of individuals in multiple environmental and social dimensions.

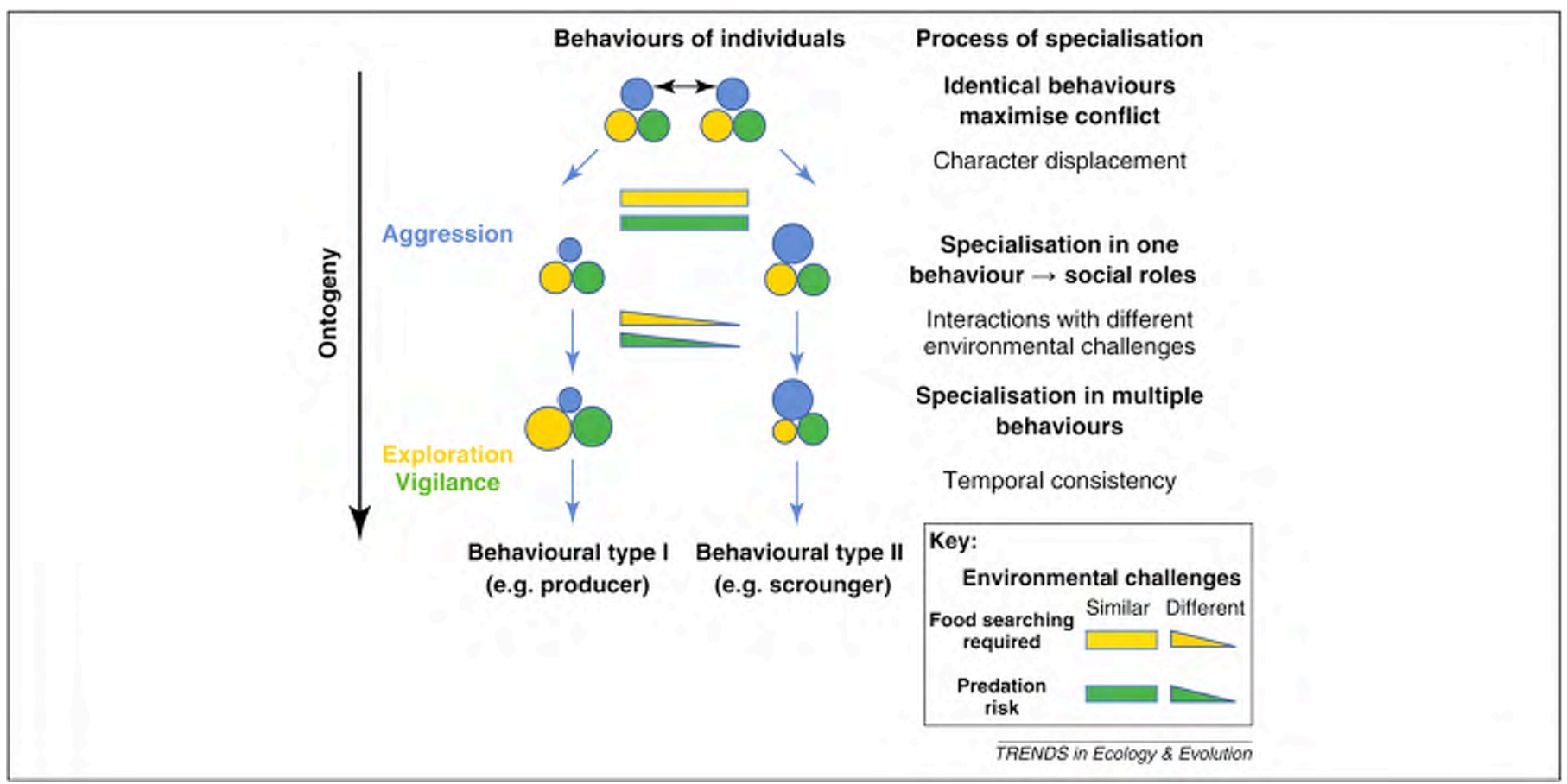

Figure 1. Strategic niche specialisation in multiple behaviours during ontogeny. Social conflict results in individuals choosing different social niches. Differently coloured circles depict the behavioural responses towards different social or ecological challenges. The behaviour of individuals (represented by groups of three circles; sizes of circles symbolise the intensity of the behaviours) is shaped by social competition and associated diverging ecological challenges. Behaviours are, for instance, aggression at food patches (blue), exploration for food (orange) and predator vigilance (green), corresponding to environmental challenges depicted in the same colours (see legend on bottom right). The right column specifies the processes in the graphical model that lead to individual specialisation in multiple behaviours. 
(b) Specialisation in a social role can affect the available options in another context. For instance, individuals might adopt a subordinate position in a hierarchy allowing them to avoid overt conflict with social partners because this is associated with less social stress [53]. Such individuals might need to be more explorative than others to obtain resources without being able to monopolise them [54], which can result in a correlation between a tendency to show submissive behaviour in encounters with group members and a proactive exploration propensity. Division of labour provides another example for how social roles might favour different packages of behaviours [38]. For instance, in cooperatively breeding species two alternative life history strategies of subordinates are the option to stay, help and queue for the breeding position, or to disperse early in order to breed independently [55]. Both options are associated with divergent social and ecological challenges and therefore should result in specific combinations of personality traits corresponding to the different life-history trajectories. A study of cooperatively breeding fish renders support for this prediction [39]. Helpers that were consistently aggressive towards intruders (a prerequisite for defending an own territory) showed low levels of territory maintenance (which only improves the natal territory), but high levels of exploration behaviour (a prerequisite for dispersal).

\section{Adaptive individual differences caused by developmental plasticity}

As with any biological feature, personality traits are produced by an interaction of genetic factors with the environment individuals encounter during different stages of life; hence, the evolution of the phenotype can be regarded as evolution of development [56]. In their developmental pathway, plastic phenotypes constantly integrate experience with their environment, which cumulatively shapes the behavioural phenotype. As the environment (social and other) is never the same for different individuals, this results in variation among individuals that is permanently screened by selection, thereby feeding back on the genotype structure in a population $[56,57]$. Hence, development (proximate causes) and selection (ultimate causes) are complementary levels explaining the observed behavioural variation [56]. Selection on phenotypic plasticity affects the evolution of reaction norms (the range of phenotypes a genotype produces in a given set of environments) [58] and the ability to learn (allowing an individual to develop an adaptive response to a novel challenge within its lifetime) [59]. Our model of developmental social specialisation (Figure 1) outlines possible mechanisms contributing to behavioural adaptation and the evolution of animal personalities based on social interactions.

\section{Social experience affecting the development of personality}

Social factors can shape personality, if experience has lasting effects on the phenotype [60-62]. For instance, in house mice early social experience (e.g. litter composition) induces changes in the behaviours of adults [63]. Personality differences have also been shown to result from sibling competition in birds $[62,64]$ and mother-offspring interactions in rodents [61] and primates [65]. This suggests that during early stages of development organisms are often flexible and susceptible to environmental influences. Environmentally mediated traits can be stabilised later in ontogeny, for instance through organisational effects of hormones or by a reduction of flexibility through learning processes with long-lasting effects [56].

Extensive studies of human personality development have investigated the puzzle of why children (including twins) within the same family often show markedly different personalities [66]. Results suggest that the shared environment (due to the same family setting) is often less important for personality development than the 'nonshared environment' resulting, for instance, from differential interactions of children among each other or with outsiders [66]. While it is difficult to determine the precise nature of the non-shared environment [67], social niche specialisation might be partly responsible for its effect. For instance, studies of monozygotic (i.e. genetically identical) conjoined (Siamese) twins often develop antithetic personalities even though they share the same environment except for the other conjoined twin [68]. When one of the two twins is extravert, open and conscientious, the other twin tends to be more introvert, shy and lackadaisical. Interestingly, the personalities of monozygotic conjoined twins differ even more from each other than those of independent monozygotic twins [68], which might exemplify the potential of niche diversification of behavioural phenotypes by social character displacement.

Developmental niche specialisation does not necessarily imply that personality prevails unchanged throughout life [60]. Instead, major transitions (e.g. in developmental stage or changes in the social environment) can lead to a reorganisation of behaviour. In humans, for example, changes in personality can still occur throughout adulthood; this potential reaches a plateau only at an age of about 50 years, after which only small changes can be detected [69].

\section{Alternative social options: animal personality and alternative tactics}

Variation in animal personality within populations can result from disruptive selection caused by social interactions, if divergent behaviour types provide higher fitness prospects than intermediate types. This process causes bior multi-modal phenotype distributions and has been studied extensively in the context of alternative behavioural and reproductive tactics [70,71]. Research in this field has focused on the question of how different solutions to the same problem can coexist within a population. For instance, intra- and inter-sexual selection can favour divergent behavioural types within a sex [34,35]. Sometimes, these differences are accompanied by morphological differences, which usually means that the (behavioural) phenotypes are fixed for life. However, this is often not the case. In fact, the vast majority of known cases of alternative tactics reflect conditional responses to momentary social conditions [72,73]. Similarly, consistent individual differences in behaviour (i.e. personality) might sometimes correspond to a diversification into discrete social roles caused by disruptive selection. For example, a dispersive role 


\section{Box 3. Social niche specialisation: implications, predictions and future directions}

There are various ways to test for the significance of social niches for the development and evolution of animal personalities. An appropriate research program might focus on the following issues (among others):

- The relationship between the proposed components of social niche specialisation should be examined:

- Niche preference: how strong is the relationship between individual predispositions, including the behavioural phenotype, and the preferred social role?

- Character displacement: what is the significance of density and frequency dependence for niche diversification of behavioural phenotypes among interacting conspecifics?

- Long-term effects of social experience: is positive feedback (e.g. effects of learning) responsible for temporal consistency of the behavioural phenotype, and are other mechanisms involved?

- Fitness consequences: how does variation in personality traits in a population affect the frequency and intensity of social conflict, and how does this affect the fitness costs of involved individuals?

- The following predictions of the concept of social niche specialisation await experimental tests:

- Consistency in behavioural differences among individuals should be greater at higher levels of social conflict, because this should reduce conflict costs (the costs of niche overlap and switching between alternatives should be higher at higher levels of conflict).

- High conflict levels should select for novel niches, i.e. social niche construction $[25,60]$.

- Social conflict concerning one context should influence behaviours expressed in other functional contexts if social specialisation affects the challenges individuals encounter in other ecological dimensions (Figure 1). Likewise, individuals pursuing alternative tactics should also differ in personality, because of the commonly diverging social and ecological challenges.

- Social niche theory of animal personality might explain why distributions of behavioural phenotypes are often continuous and unimodal: multidimensional social niche options might select for fine-tuned specialisations due to multi-niche frequency dependence. Instead, the bi- or multi-modal trait distributions characterising species exhibiting alternative tactics hint at disruptive selection in mainly one ecological dimension (e.g. [50]). This potential difference between the occurrence of animal personalities and distinctive alternative tactics should be addressed in future studies.

- Studies of animal personality and alternative tactics would both benefit from a better understanding of potential threshold mechanisms and developmental switches responsible for the generation of diverging behavioural profiles. might require a high exploration propensity, while a philopatric role might be better associated with a low tendency to explore. Likewise, resource monopolisation requires high aggressiveness levels, while social parasitism might favour submissiveness. In these and other contexts, intermediate types might yield lower fitness because they perform worse than the 'specialists' in either situation. In other words, disruptive selection could favour that individuals occupying certain social roles are either aggressive or reserved, responsive or unresponsive, explorative or contained, pro-active or reactive, while behavioural 'generalists' might be penalised. However, as yet research on animal personality has given little attention to the distribution of behavioural phenotypes within populations. Hence it is unknown in most cases whether personality types show a uni-, bi- or multi-modal distribution in a population, and the potential role of disruptive selection is obscure.

It is conceivable that similar evolutionary and developmental processes are involved in the generation of alternative tactics and personality variation. In principle, selection for polymorphic characters involves threshold traits [74]. These reflect the effects of quantitative trait loci, where phenotype expression depends on whether a 'liability' value passes a threshold [75]. This has, for example, been demonstrated for the expression of aggressive and non-aggressive male morphs in mites, in which the threshold reaction norm was shifted by selection experiments [76]. Phenotype expression depending on thresholds might also occur when changes of personality traits result from particular types of experience exceeding a threshold (e.g. traumatic effects, early social effects or long term experience) [60]. Threshold traits often operate during development, with abrupt changes between alternative pathways at particular points, e.g. at a particular body size, producing different phenotypes on either side of the threshold [77]. Hence, threshold traits could gener- ate lasting environmental effects on personality during development, thereby triggering the expression of alternative life-history strategies. As developmental thresholds have a genetic basis, trait expression is both conditional and heritable, allowing alternative phenotypes to evolve largely independently from each other [35]. This greatly increases the scope for the evolution of different phenotypes [56]. Importantly, the thresholds or developmental switch points involved in phenotype determination are subject to selection and adaptive evolution due to their genetic basis [78]. The potential similarities and overlap of mechanisms responsible for the evolution of animal personality and alternative tactics suggest that a stronger link between research on animal personality and concepts developed in the study of alternative tactics would be worthwhile (Box 3).

\section{Conclusions}

The framework of social niche specialisation provides an adaptive explanation for the existence of animal personality differences among individuals in a social context based on the dynamic effects of interactions between individuals throughout life. It rests on the assumption that individuals increase their fitness by choosing behavioural strategies that reduce conflict with other members of the same population. Selection should favour traits providing effective solutions for social conflict. Behavioural consistency might serve to diminish conflict among conspecifics because it reduces niche overlap between individuals using the same resources, which is arguably the most important source of social conflict.

\section{Acknowledgements}

The preparation of this manuscript was supported by the Swiss National Science Foundation (grant 3100A0-108019 to Redouan Bshary and grant 3100A0-122511 to MT). We thank R. Bshary, Ian Hamilton, Dik Heg, Laurent Lehmann, Barbara Taborsky, and the students and colleagues from the Behavioural Ecology group at the University of Bern and from 
the Eco-Ethology group at the University of Neuchâtel for discussion; and Ian Hamilton, Katharina Peer, Roger Schürch, Dolores Schütz and anonymous referees for helpful comments on earlier versions of the manuscript.

\section{References}

1 Gosling, S.D. (2001) From mice to men: what can we learn about personality from animal research? Psychol. Bull. 127, 45-86

2 Sih, A. et al. (2004) Behavioral syndromes: an ecological and evolutionary overview. Trends Ecol. Evol. 19, 372-378

3 Dingemanse, N.J. and Réale, D. (2005) Natural selection and animal personality. Behaviour 142, 1159-1184

4 Bell, A. (2007) Future directions in behavioural syndromes research. Proc. R. Soc. Lond. B 274, 755-761

5 Reale, D. et al. (2007) Integrating animal temperament within ecology and evolution. Biol. Rev. Camb. Philos. Soc. 82, 291-318

6 Koolhaas, J.M. et al. (1999) Coping styles in animals: current status in behavior and stress-physiology. Neurosci. Biobehav. Rev. 23, 925935

7 Bergmüller, R. (2010) Animal personality and behavioural syndromes. In Animal Behaviour - Evolution and Mechanisms (Kappeler, P., ed.), pp. 587-621, Springer

8 Wolf, M. et al. (2008) Evolutionary emergence of responsive and unresponsive personalities. Proc. Natl. Acad. Sci. U. S. A. 105, $15825-15830$

9 Biro, P.A. and Stamps, J.A. (2008) Are animal personality traits linked to life-history productivity? Trends Ecol. Evol. 23, 361-368

10 Dingemanse, N.J. et al. (2007) Behavioural syndromes differ predictably between 12 populations of three-spined stickleback. J. Anim. Ecol. 76, 1128-1138

11 Dall, S.R.X. et al. (2004) The behavioural ecology of personality: consistent individual differences from an adaptive perspective. Ecol. Lett. 7, 734-739

12 Schuett, W. et al. (2010) Sexual selection and animal personality. Biol. Rev. Camb. Philos. Soc. 85, 217-246

13 Reale, D. and Dingemanse, N.J. (2010) Personality and individual social specialisation. In Social Behaviour: Genes Ecology and Evolution (Szelely, T. et al., eds), Cambridge University Press

14 Bergmüller, R. et al. (2010) Evolutionary causes and consequences of consistent individual variation in cooperative behaviour. Phil. Trans. R. Soc. Lond. B DOI: 10.1098/rstb.2010.0124

15 Hutchinson, G.E. (1959) Homage to Santa-Rosalia or why are there so many kinds of animals. Am. Nat. 93, 145-159 DOI: 10.1086/282070

16 Begon, M. et al. (2005) Ecology: From Individuals to Ecosystems, (4th edition), Wiley-Blackwell

17 Grant, B.R. and Grant, P.R. (2003) What Darwin's finches can teach us about the evolutionary origin and regulation of biodiversity. Bioscience 53，965-975 doi:10.1641/0006-3568(2003)053[0965: WDFCTU]2.0.CO;2

18 Alatalo, R.V.et al. (1987) Exploitation competition influences the use of foraging sites by tits: experimental evidence. Ecology 68, 284-290

19 Wilson, D.S. (1998) Adaptive individual differences within single populations. Phil. Trans. R. Soc. Lond. B 353, 199-205

20 Buss, D.M. (1991) Evolutionary personality psychology. Annu. Rev. Psychol. 42, 459-491

21 Buss, D.M. (1990) Biological foundations of personality evolution, behavioral-genetics and psychophysiology - toward a biologically informed psychology of personality. J. Pers. 58, 1-16

22 Bolnick, D.I. et al. (2003) The ecology of individuals: Incidence and implications of individual specialization. Am. Nat. 161, 1-28

23 Gosling, S.D. and John, O.P. (1999) Personality dimensions in nonhuman animals: A cross-species review. Curr. Dir. Psychol. Sci. $8,69-75$

24 Penke, L. et al. (2007) The evolutionary genetics of personality. Eur. J. Pers. 21, 549-587

25 Plomin, R. et al. (1977) Genotype environment interaction and correlation in analysis of human behavior. Psychol. Bull. 84, 309-322

26 Wolf, J.B. et al. (1999) Interacting phenotypes and the evolutionary process. II. Selection resulting from social interactions. Am. Nat. 153, 254-266

27 Parker, G.A. (1979) Sexual selection and sexual conflict. In Sexual Selection and Reproductive Competition in Insects (Blum, M.S. and Blum, N.A., eds), pp. 123-166, Academic Press
28 Trivers, R.L. (1974) Parent offspring conflict. Am. Zool. 14, 249-264

29 Trivers, R.L. (1972) Parental investment and sexual selection. In Sexual Selection and the Descent of Man (Campbell, B., ed.), pp. 1871-1971, Chicago, Aldine Transaction

30 van Schaik, C.P. (1989) The ecology of social relationships among female primates. In Comparative Socioecology, the Behavioural Ecology of Human and Other Primates (Standen, V. and Fole, R.A., eds), pp. 195-218, Blackwell

31 Barnard, C.J. and Sibly, R.M. (1981) Producers and scroungers: A general-model and its application to captive flocks of house sparrows. Anim. Behav. 29, 543-550

32 Giraldeau, L.A. and Beauchamp, G. (1999) Food exploitation: searching for the optimal joining policy. Trends Ecol. Evol. 14, 102-106

33 Giraldeau, L.A. and Dubois, F. (2008) Social foraging and the study of exploitative behavior. In Advances in the Study of Behavior, Vol 38, pp. 59-104

34 Taborsky, M. et al. (2008) The evolution of alternative reproductive tactics: concepts and questions. In Alternative Reproductive Tactics: an Integrative Approach (Oliveira, R. et al., eds), pp. 1-21, Cambridge University Press

35 Taborsky, M. and Brockmann, H.J. (2010) Alternative reproductive tactics and life history phenotypes. In Animal Behaviour: Evolution and Mechanisms (Kappeler, P., ed.), pp. 537-586, Springer

36 Maynard Smith, J. (1982) Evolution and the Theory of Games, Cambridge University Press

37 Wilson, D.S. et al. (1994) Shyness and boldness in humans and other animals. Trends Ecol. Evol. 9, 442-446 DOI: 10.1016/01695347(94)90134-1

38 Beshers, S.N. and Fewell, J.H. (2001) Models of division of labor in social insects. Annu. Rev. Entomol. 46, 413-440

39 Bergmüller, R. and Taborsky, M. (2007) Adaptive behavioural syndromes due to strategic niche specialization. BMC Ecol. 7, 12 DOI: $10.1186 / 1472-6785-7-12$

40 Holbrook, C.T. et al. (2009) Emergence and consequences of division of labor in associations of normally solitary sweat bees. Ethology 115, 301-310 DOI: 10.1111/j.1439-0310.2009.01617.x

41 Jeanson, R. et al. (2008) Division of labour and socially induced changes in response thresholds in associations of solitary halictine bees. Anim. Behav. 76, 593-602 DOI: 10.1016/j.anbehav.2008.04.007

42 Bolnick, D.I. (2001) Intraspecific competition favours niche width expansion in Drosophila melanogaster. Nature 410, 463-466

43 DeWitt, T.J. et al. (1998) Costs and limits of phenotypic plasticity. Trends Ecol. Evol. 13, 77-81 DOI: 10.1016/S0169-5347(97)01274-3

44 Sapolsky, R.M. et al. (2000) How do glucocorticoids influence stress responses? Integrating permissive, suppressive, stimulatory, and preparative actions. Endocr. Rev. 21, 55-89

45 Monsell, S. (2003) Task switching. Trends Cogn. Sci. 7, 134-140

46 Bergmüller, R. et al. (2005) Extended safe havens and between group dispersal of helpers in a cooperatively breeding cichlid. Behaviour 142, 1643-1667 DOI: 10.1163/156853905774831800

47 Morand-Ferron, J. and Giraldeau, L.A. (2010) Learning behaviorally stable solutions to producer-scrounger games. Behav. Ecol. 21, 343-348 DOI: $10.1093 /$ beheco/arp195

48 Lande, R. and Arnold, S.J. (1983) The measurement of selection on correlated characters. Evolution 37, 1210-1226

49 Brodie, E.D. (1992) Correlational selection for color pattern and antipredator behavior in the garter snake Thamnophis ordinoides. Evolution 46, 1284-1298

50 Eaves, L.J. et al. (1990) Personality and reproductive fitness. Behav. Genet. 20, 563-568

51 Stirling, D.G. et al. (2002) Selection, structure and the heritability of behaviour. J. Evol. Biol. 15, 277-289 DOI: 10.1046/j.14209101.2002.00389.x

52 Cote, J. and Clobert, J. (2007) Social personalities influence natal dispersal in a lizard. Proc. R. Soc. Lond. B 274, 383-390

53 Sapolsky, R.M. (2005) The influence of social hierarchy on primate health. Science $308,648-652$

54 Liker, A. and Barta, Z. (2002) The effects of dominance on social foraging tactic use in house sparrows. Behaviour 139, 1061-1076

55 Cahan, S.H. et al. (2002) Social trajectories and the evolution of social behavior. OIKOS 96, 206-216

56 West-Eberhard, M.J. (2003) Developmental Plasticity and Evolution, Oxford University Press 
57 Pigliucci, M. et al. (2006) Phenotypic plasticity and evolution by genetic assimilation. J. Exp. Biol. 209, 2362-2367

58 Dingemanse, N.J. et al. (2010) Behavioural reaction norms: animal personality meets individual plasticity. Trends Ecol. Evol. 25, 81-89

59 Paenke, I. et al. (2007) Influence of plasticity and learning on evolution under directional selection. Am. Nat. 170, E47-E58

60 Stamps, J. and Groothuis, G.G. (2010) The development of animal personality: relevance, concepts and perspectives. Biol. Rev. Camb. Philos. Soc. 58, 301-325

61 Meaney, M. (2001) Maternal care, gene expression, and the transmission of individual differences in stress reactivity across generations. Annu. Rev. Neurosci. 24, 1161-1192

62 Groothuis, T.G.G. and Carere, C. (2005) Avian personalities: characterization and epigenesis. Neurosci. Biobehav. Rev. 29, 137-150

63 Benus, R.F. and Henkelmann, C. (1998) Litter composition influences the development of aggression and behavioural strategy in male Mus domesticus. Behaviour 135, 1229-1249

64 Carere, C. et al. (2005) Epigenetic effects on personality traits: early food provisioning and sibling competition. Behaviour 142, 1329-1355 DOI: $10.1163 / 156853905774539328$

65 Coplan, J.D. et al. (1996) Persistent elevations of cerebrospinal uid concentrations of corticotropin-releasing factor in adult nonhuman primates exposed to early-life stressors: Implications for the pathophysiology of mood and anxiety disorders. Proc. Natl. Acad. Sci. U. S. A. 93, 1619-1623

66 Plomin, R. and Daniels, D. (1987) Why are children in the same family so different from each other? Behav. Brain Sci. 10, 1-16 DOI: 10.1017/ S0140525X00055941

67 Plomin, R. et al. (2001) Why are children in the same family so different? Nonshared environment a decade later. Can. J. Psychiatry 46, 225-233

68 Smith, J.D. (1988) Psychological Profiles of Conjoined Twins: Heredity, Environment, and Identity, Greenwood Publishing Group, Praeger Publishers

69 Caspi, A. et al. (2005) Personality development: Stability and change. Annu. Rev. Psychol. 56, 453-484

70 Brockmann, H.J. and Taborsky, M. (2008) Alternative reproductive tactics and the evolution of alternative allocation phenotypes. In Alternative Reproductive Tactics: an Integrative Approach ((Oliveira, R. et al., eds), pp. 25-51, Cambridge University Press

71 Brockmann, H.J. et al. (2008) Integrating mechanisms and function: prospects for future research. In Alternative Reproductive Tactics: an Integrative Approach (Oliveira, R. et al., eds), pp. 471-489, Cambridge University Press

72 Gross, M.R. (1996) Alternative reproductive strategies and tactics: Diversity within sexes. Trends Ecol. Evol. 11, 92-98

73 Oliveira, R.F. et al., eds (2008) Alternative Reproductive Tactics. An Integrative Approach, Cambridge University Press

74 Roff, D.A. (1996) The evolution of genetic correlations: An analysis of patterns. Evolution 50, 1392-1403

75 Falconer, D.S. and Mackay, T.F.C. (1996) Introduction into Quantitative Genetics, Longman
76 Unrug, J. et al. (2004) Alternative phenotypes and sexual selection: can dichotomous handicaps honestly signal quality? Proc. R. Soc. Lond. B $271,1401-1406$

77 Emlen, D.J. and Nijhout, H.F. (2000) The development and evolution of exaggerated morphologies in insects. Annu. Rev. Entomol. 45, 661708

78 Tomkins, J.L. and Brown, G.S. (2004) Population density drives the local evolution of a threshold dimorphism. Nature 431, 10991103

79 Brown, W.L. and Wilson, E.O. (1956) Character displacement. Syst. Zool. 5, 49-65 DOI: 10.2307/2411924

80 Aureli, F. and de Waal, F.B.M. (2000) Why natural conflict resolution? In Natural Conflict Resolution (Aureli, F. and de Waal, F.B.M., eds), pp. 3-10, University of California Press Ltd

81 Sih, A. and Bell, A.M. (2008) Insights for behavioral ecology from behavioral syndromes. In Advances in the Study of Behavior, Vol 38, pp. 227-281

82 Bell, A.M. et al. (2009) The repeatability of behaviour: a meta-analysis. Anim. Behav. 77, 771-783 DOI: 10.1016/j.anbehav.2008.12.022

83 Drent, P.J. et al. (2003) Realized heritability of personalities in the great tit. Proc. R. Soc. Lond. B 270, 45-51

84 Stamps, J.A. (2007) Growth-mortality tradeoffs and 'personality traits' in animals. Ecol. Lett. 10, 355-363

85 Wolf, M. et al. (2007) Life-history trade-offs favour the evolution of animal personalities. Nature 447, 581-585

86 Bell, A.M. and Sih, A. (2007) Exposure to predation generates personality in threespined sticklebacks (Gasterosteus aculeatus). Ecol. Lett. 10, 828-834

87 Michelena, P.et al. (2009) Effects of group size and personality on social foraging: the distribution of sheep across patches. Behav. Ecol. 20, 145152 DOI: 10.1093/beheco/arn126

88 Arnold, C. and Taborsky, B. (2010) Social experience in early ontogeny has lasting effects on social skills in cooperatively breeding cichlids. Anim. Behav. 79, 621-630 DOI: 10.1016/j.anbehav.2009.12.008

89 Slagsvold, T. and Wiebe, K.L. (2007) Learning the ecological niche. Proc. R. Soc. Lond. B 274, 19-23

90 D’Eath, R.B. and Burn, C.C. (2002) Individual differences in behaviour: A test of 'coping style' does not predict resident-intruder aggressiveness in pigs. Behaviour 139, 1175-1194

91 Dyer, J.R.G. et al. (2009) Shoal composition determines foraging success in the guppy. Behav. Ecol. 20, 165-171 DOI: 10.1093/beheco/ $\operatorname{arn} 129$

92 Grasmuck, V. and Desor, D. (2002) Behavioural differentiation of rats confronted to a complex diving-for-food situation. Behav. Processes 58, 67-77

93 Frost, A.J. et al. (2007) Plasticity in animal personality traits: does prior experience alter the degree of boldness? Proc. R. Soc. Lond. B 274, 333-339

94 Chapman, B.B. et al. (2008) Schooling and learning: early social environment predicts social learning ability in the guppy, Poecilia reticulata. Anim. Behav. 76, 923-929 DOI: 10.1016/j.anbehav. 2008.03.022 